\title{
An Assessment of Reprocessed GPS/MET Observations Spanning 1995-1997
}

Anthony J. Mannucci ${ }^{1}$, Chi Ao ${ }^{1}$, Byron A. Iijima ${ }^{1}$, Thomas K. Meehan ${ }^{1}$, Panagiotis $\operatorname{Vergados}^{1}$, E. Robert Kursinski ${ }^{2}$, Sean Healy ${ }^{3}$, Andras Horányi ${ }^{3}$, William S. Schreiner ${ }^{4}$

$5 \quad{ }^{1}$ Jet Propulsion Laboratory, California Institute of Technology, Pasadena, CA, 91109, USA

${ }^{2}$ PlanetiQ, Golden, CO, 80401, USA

${ }^{3}$ European Centre for Medium-Range Weather Forecasts, Reading, RG2 9AX, United Kingdom

${ }^{4}$ University Corporation for Atmospheric Research, Boulder, CO, 80307, USA

Correspondence to: Anthony J. Mannucci (anthony.j.mannucci@jpl.nasa.gov)

10 Abstract. We have performed an analysis of reprocessed GPS/MET data spanning 1995-1997 generated by CDAAC in 2007. CDAAC developed modified dual-frequency processing methods for the encrypted data (AS-on) during 1995-1997. We compared the CDAAC data set to the MERRA-2 reanalysis, separately for AS-on and AS-off, focusing on the altitude range 10-30 km. MERRA-2 did not assimilate GPS/MET data in the period 1995-1997. To gain insight into the CDAAC data set, we developed a single-frequency data set for GPS/MET, which is unaffected by the presence of encryption. We find

15 excellent agreement between the more limited single frequency data set and the CDAAC data set: the bias between these two data sets is consistently less than $0.25 \%$ in refractivity, whether or not AS is on. Given the different techniques applied between the CDAAC and JPL data sets, agreement suggests that the CDAAC AS-on processing and the single frequency processing are not biased in an aggregate sense greater than $0.25 \%$ in refractivity, which corresponds approximately to a temperature bias less than $0.5 \mathrm{~K}$. Since the profiles contained in the new single frequency data set are not a subset of the CDAAC profiles, the combination of the CDAAC data set, consisting of 9,579 profiles, and the new single-frequency data set, consisting of 4,729 profiles, yields a total number of 11,531 unique profiles from combining the JPL and CDAAC data sets. All numbers are after quality control has been applied by the respective processing activities. 


\section{Introduction}

There is currently intense interest in Earth science observations that are useful for measuring decadal scale changes to the climate (Wielicki et al., 2013). When considering such observations from orbiting platforms, measurement accuracy needs to be characterized over time scales that exceed the lifetime of any single mission (Gleisner et al., 2020). Therefore, combining observations from multiple missions and understanding their changing properties over several years must be addressed.

The Global Navigation Satellite System (GNSS) radio occultation (RO) technique has the potential to provide such long-

40 term observations with accuracies that are sufficient to meet the stringent demands of climate change observation, documented as $0.05 \mathrm{~K}$ temperature stability per decade (Leroy et al., 2006; Ho et al., 2009; Steiner et al., 2013; GCOS, 2016). GNSS RO has been used in climate trend studies that use climate model finger-printing techniques (Lackner et al., 2011) or the observations directly (Steiner et al., 2009; Steiner et al., 2020a; Vergados et al., 2021). A significant challenge in observing decadal-scale climate change is that geophysical variables display significant variation on time scales shorter

45 than tens of years. Such "natural variability" is potentially the most significant factor hindering the interpretation of observations relevant to climate change (Leroy et al., 2008; Santer et al., 2017). It is widely recognized that the longer a time series is, the more valuable it is for observing climate change (Leroy et al., 2008; Wielicki et al., 2013).

In this paper, we analyze observations from the RO record dating back to 1995 when the first observations were obtained

50 from the "proof-of-concept" GPS/MET mission (Kursinski et al., 1996; Rocken et al., 1997). GPS/MET data have been used successfully in climate-related studies, but generally using only a limited subset of the acquired data consisting of two threeweek periods when the data quality was highest (Oct 1995 and Feb 1997) (Steiner et al., 2009; Steiner et al., 2011). There exists a much more extensive data set from 2007 that includes many more months in the period 1995-1997 that has not yet been evaluated in the literature but that could contribute to more robust trend detection.

The two limited GPS/MET periods coincide with two favorable conditions that contributed to increased data quality: 1) lack of encryption of the second transmitted GPS frequency (L2, $1227.60 \mathrm{MHz}$ ), thus permitting nominal signal-to-noise ratios (SNR) for processing and 2) favorable orientation of the spacecraft permitting the aft-directed antenna to be aligned with the velocity direction of the spacecraft (so-called "prime" periods; Schreiner et al., 1998). Signal encryption is also known as "Anti-Spoofing" (AS). In this paper, we distinguish periods when encryption was active, also called "AS-on", from periods when encryption was temporarily disabled (“AS-off”). Prime periods, which had the favorable antenna orientation, could occur when encryption was on or off. Throughout the GPS/MET mission, one or the other or both of these conditions periodically occurred. 
65 Past climate studies have tended to use data only from the periods when both the favorable antenna orientation and AS-off were present. Such periods permit standard dual-frequency processing of the observations, which is used to reduce the impact of ionospheric delays on the retrievals (Rocken et al., 1997; Hajj et al., 2002). There are two published studies using data with an encrypted second frequency and thus reduced signal-to-noise ratios (SNRs) (Nishida et al., 2000; Randel et al. 2003). Randel et al. (2003) noted no discernable difference comparing periods with and without encryption. However, these studies did not use the data to analyze trends, and they did not use the reprocessed data set from the University Corporation for Atmospheric Research (UCAR) COSMIC Data Analysis and Archive Center (CDAAC) published in 2007. We note that all studies to date (including this one) use data only from "prime" periods of favorable spacecraft orientation.

The main distinguishing feature of this study versus past studies using GPS/MET is a detailed comparison with a reanalysis clearly distinguishing periods when anti-spoofing encryption was turned on versus off. The AS-on period resulted in significantly reduced L2 SNRs that required additional temporal smoothing to be applied to this frequency. This necessitated modified processing techniques that were finalized for the 2007 data set but remained a concern for users of the data interested in climate trends or for evaluation (Steiner et al., 2009; Lackner et al., 2011; Marquardt et al., 2001; Gorbunov and Kornblueh, 2001). UCAR's CDAAC generated such a re-processed data set in 2007 encompassing 9,579 profiles spanning 1995-1997 that has yet to be fully characterized for scientific use. JPL also produced a dual-frequency data set during this period, although it will not be evaluated for this paper.

Recent interest in the potential of using reanalyses for climate trend studies (Bosilovich et al., 2015) motivate interest in these RO data sets from GPS/MET because they extend over the period which reanalyses can be evaluated, and may provide insight into how a uniformly processed reanalysis might be subject to biases that change over time, particularly going back to the 1990s when fewer space-based observations were available than currently. Assessing the structural uncertainty of RO measurements is a major activity (Ho et al., 2009; Ho et al., 2012; Steiner et al., 2013; Steiner et al., 2020b) reflecting that, despite an origin of common raw data, the processing chains of different centers produce different results due to reasonable but different processing choices and algorithms adopted by those centers.

90

It is the purpose of this work to provide insights on the magnitudes of possible biases of the CDAAC re-processed data set when encryption is present by comparing it with a new data set produced at JPL using single frequency processing. As a corollary benefit, an expanded data set is now available from the period based on these new reprocessed data. As is typical for RO data sets (Ho et al., 2012; Steiner et al., 2020b), different processing strategies that use different methods of quality 95 control do not reject the same set of profiles, possibly indicating the quality control criteria are too strict. The single frequency processing strategy described in this paper is not susceptible to limitations of encryption that are applied to the L2 frequency during periods of AS-on. In this work, we compare the CDAAC dual-frequency data set with new JPL single frequency retrievals during conditions when AS is on or off. We also compare to the MERRA-2 reanalysis (Basiliovich et 
al., 2015), a reanalysis spanning 1980 to the present, produced by NASA's Global Modeling and Assimilation Office. MERRA-2 does not assimilate RO data from 1995-1997, so it can be considered an independent source of information about potential biases in GPS/MET data. However, MERRA-2 may itself be biased. An additional "third vote" data set, which is available from single-frequency processing, provides clarity on what potential biases might be. This is similar in spirit to the three-corner hat technique applied by Anthes and Rieckh (2018) which is used to characterize noise in four data sets.

In this work, we present an analysis of two reprocessed GPS/MET data sets that have so far not been assessed in the literature to our knowledge. Section 2 describes the data sets available for analysis, including a description of how they were obtained compared to standard dual-frequency processing. In Section 3 we present the results of an assessment that compares the two data sets to the MERRA-2 reanalysis product and to each other (CDAAC and JPL). In Section 4 we discuss the results and what they may imply about biases in the GPS/MET record and in the reanalysis. We conclude in Section 5 and provide suggestions for further research.

\section{Data Sets}

GPS/MET data were collected over each month spanning 1995-1997 (Rocken at al., 1997). Assessing the data from the full GPS/MET data set is valuable as a means of assessing reanalyses during the period 1995-1997 or as a prelude to assimilating these data in future reanalysis activities. Reanalysis biases in the upper troposphere/lower stratosphere are shown to be

115 reduced when radio occultation data are assimilated (Poli et al., 2010; Ruston and Healy, 2020).

A reprocessing was performed at CDAAC in 2007. Figure 1 shows statistics from the data set, including global coverage and the number of profiles by month currently available in the CDAAC archive. All data shown are after quality control has been applied. To our knowledge, an analysis of the data reprocessed in 2007 has not been published. The reprocessing used a

120 similar software base to what was used for the COSMIC data set (Schreiner et al., 1998). COSMIC launched in April, 2006 and underwent reprocessing in 2007.

The new data set introduced in this paper is a re-processed data set from JPL using a single-frequency processing approach applied to the occulting link from which the atmospheric refractivity information is obtained. A benefit of the single-

125 frequency data set is to assess the dual-frequency data set when the second GPS frequency was encrypted (AS-on) that required a non-standard processing method to be adopted for the L2 frequency. Single frequency processing does not use the L2 frequency, so potential biases introduced by the non-standard processing may be revealed by comparing the two data sets. Results of these comparisons are discussed in Section 3. 
Despite its lower number of profiles, the single frequency data set presented here is not a subset of the CDAAC data set, so combining the CDAAC data set with this new data set will increase the overall number of available profiles from GPS/MET. There is also a dual-frequency data set from JPL that covers both AS-on and AS-off periods. We will not be assessing that data set in this paper. We emphasize that the CDAAC and JPL refractivity data sets presented in this paper are independently processed starting with the raw data, including independent orbit determination for GPS/MET and clock calibrations.

\subsection{Single-Frequency Processing}

It has been recognized since the earliest development of GNSS radio occultation that signal delays caused by the Earth's ionosphere must be calibrated to achieve sufficiently accurate results in the neutral atmosphere (Kursinski et al., 1996). This calibration is usually achieved using the two frequencies that are broadcast by GNSS systems specifically to minimize the influence of the ionized upper atmosphere on precise positioning applications. However, it has been widely reported that dual-frequency calibration methods do not fully remove ionospheric effects (Vorob'ev and Krasil'nikova, 1994; Kursinski et al., 1997). Significant effort has been expended to understand the impact of residual ionospheric delay biases introduced in the upper troposphere and lower stratosphere (Syndergaard, 2000; Steiner and Kirchengast, 2005; Mannucci, et al., 2011; Vergados et al., 2011; Danzer et al., 2013; Danzer et al., 2020). Single-frequency processing is subject to different residual effects, primarily due to multi-path on the pseudorange signal (discussed below), so comparison between single and dualfrequency processing is another means of assessing residual biases. In general, and particularly for the more recent generation of GNSS receivers, single frequency processing is expected to have lower precision than dual-frequency processing due to the use of the much noisier pseudorange. However, for climate studies, the precision is less important than biases that may appear between the daytime and nighttime profiles due to ionospheric effects.

The single frequency retrieval processing used in this paper is described next. The foundation of the method is to use the difference between phase and group delays of the transmitted signal, taking advantage of the fact that phase and group delay in the dispersive ionospheric medium are of equal but opposite sign (Davies, 1990). Single frequency radio occultation processing has been described previously in two publications (de la Torre et al., 2004; Larsen et al, 2005). Following a notation similar to that used in de la Torre et al. (2004), we can write the phase delay $L_{k}$ of a calibrated GPS signal as:

$$
L_{k}=\eta-\frac{I}{f_{k}^{2}}+v_{k}
$$

where $\eta$ is the (non-dispersive) atmospheric delay, $I$ is an ionospheric delay contribution proportional to the column density of electrons along the transmitter-receiver raypath (total electron content), and $v_{k}$ is the phase noise contribution due to thermal and multipath sources. The index $k$ specifies the transmission frequency $f_{k}(=1575.42 \mathrm{MHz}$ for $k=1$ and 1227.60 $\mathrm{MHz}$ for $k=2$ ). The signal here is assumed calibrated in the sense that geometric delays and clock biases are assumed known and removed. The group delay of the signal $P_{k}$ derived from the GPS ranging code is: 


$$
P_{k}=\eta+\frac{I}{f_{k}^{2}}+\mu_{k}
$$

where $\mu_{k}$ is the noise associated with the ranging signals. Due to the much longer wavelength of the ranging code compared to the phase (300 m versus $19 \mathrm{~cm}$ for phase) the range precision is much less than the phase precision, and the multipath noise is larger. That is why the group delay is generally not used in radio occultation. However, if only a single frequency is available, a combination of the range and phase delays yields a biased estimate of the ionospheric delay $I$, that can then be used to correct the phase delay at the available $f_{1}$ frequency (also called L1) to recover the atmospheric delay $\eta$ using Equation (1). The range and phase combination is given by:

$$
I=0.5 f_{1}^{2}\left(P_{1}-L_{1}+\mu_{1}-v_{1}\right)
$$

170 The occultation retrieval proceeds as usual after subtracting the ionospheric delay calculated as above from the phase delay at the L1 frequency. We term this approach "direct phase correction" because the phase is adjusted before deriving bending angles. This differs from the standard dual-frequency approach that applies the dual-frequency correction to bending angles rather than to the signal phase (Syndergaard, 2000). The standard ionospheric correction in bending angle space, at common impact parameter, is meant to compensate for the raypath separation that occurs between the two frequencies L1 and L2.

175 Raypath separation is much less in the single frequency approach and will be ignored here, because the bandwidth of the precise ranging code $\mathrm{P}$-code $(10 \mathrm{MHz})$ is much less than the frequency separation between the $\mathrm{L} 1$ and $\mathrm{L} 2$ frequencies $(347.8$ $\mathrm{MHz})$.

As discussed in de la Torre et al. (2004), there are two raypaths for which an ionospheric delay estimate is required: the occulting link, and the non-occulting link used for calibrating the receiver clock. Since the non-occulting link is affected by ionospheric delay only above satellite altitude $(\sim 730 \mathrm{~km})$, its ionospheric delay is much less than on the occulting link which propagates through the maximum region of the electron density profile. Correspondingly, the delay change of the nonocculting link over the occultation time of $\sim 120$ seconds is much less for the non-occulting link as the ionosphere tends to vary more smoothly at altitudes above the satellite. Therefore, the single-frequency correction was only applied to the occulting link in this work. The calibration links used two frequencies, despite that the L2 frequency was much weaker, because smoothing can be applied effectively when the ionosphere does not vary rapidly. During the GPS/MET experiment, additional calibration links are required to calibrate the GPS clocks, since they were intentionally dithered using a technique called "selective availability". As with the calibration link, the ionospheric delay for these ground-based links vary negligibly during the occultations, and the L2 frequency can be smoothed without incurring significant error. 
Deriving the ionospheric correction from Equation (3) requires several considerations. First, the pseudorange estimate $P_{1}$ is available at 1 -second intervals, whereas the phase data $L_{1}$ are produced at a $50 \mathrm{~Hz}$ rate. Therefore, a means is required to derive $I$ at $50 \mathrm{~Hz}$. Second, the random errors in the pseudorange data are at least a factor of 100 larger than in the phase data. To produce retrieval profiles with similar random uncertainties to the dual-frequency technique requires that temporal smoothing be applied directly to $P_{1}$. The smoothing algorithm to reduce the pseudorange noise is critical. As has been shown by von Engeln et al. (2009), smoothing algorithms themselves can introduce biases in the data that depend on the level of noise, so care is required to minimize smoothing-related biases for climate applications.

The theoretical basis for smoothing the ionospheric delay time series is that the ionospheric delay contribution to the phase varies slowly compared to the noise introduced by the pseudorange. We assume that the slow ionospheric variation is described by a series of low-order polynomials over the altitude range of the occultation, although we do not know in advance what polynomial change in delay is caused by the ionosphere. We therefore use standard least squares methods to estimate the underlying polynomial. This requires that we assume a polynomial order for the fit (linear, quadratic, etc.). The assumption that the ionospheric contribution to phase delay is slowly varying is valid in the absence of small-scale ionospheric irregularities (Hajj et al., 2002) that cause phase scintillation. In the presence of irregularities, other means are required to assess the potential bias such irregularities may impose (Verkhoglyadova et al., 2015). We do not further consider the potential impact of ionospheric irregularities on the ionospheric correction.

When considering a polynomial-based temporal smoothing algorithm applied to the time series $P_{1}$, it is clearly advantageous to fit the polynomial over as limited a time range as possible, taking advantage of Taylor's theorem asserting that smooth functions (in this case, the ionospheric delay versus time) are well approximated by low-order polynomials over sufficiently limited time intervals. It is also advantageous to use low-order polynomials to avoid fitting to the noise. However, for a given polynomial fit order, lesser time intervals for the fitting lead to less smoothing and less filtering of the noise. On the other hand, fitting over too long a time interval will introduce biases if the underlying ionospheric variation over time is not well described by the low order polynomial used in the fit.

The approach used by de la Torre et al. (2004), of which a variant is used here, is to fit polynomials separately over several temporal sub-intervals during the occultation. The smoothed value is recovered from the center of each fit, moving forward in time, except at the edges, where the most central point possible is used. Fitting the polynomial over a limited portion of the radio occultation period reduces biases associated with choosing a low-order polynomial in the fitting.

The variant to this sliding time window approach that we use here is to combine estimates from multiple fitting sub-intervals, rather than use only the estimate from one sub-interval. Combining multiple fits reduces the variance of the smoothed result, however it averages together quantities that have correlated noise. The average of quantities with correlated noise is not a 
zero-mean estimator of the underlying quantity to which the noise is added. Thus, a bias might be introduced in this averaging, even as the variance is reduced. However, the bias introduced in this way will not be the same from profile to profile and is unlikely to result in climatological bias when multiple profiles are averaged.

The smoothing approach is illustrated in Figure 2. In the upper part of the figure, the "fitting step" is described. The time domain of the occultation is partitioned into multiple overlapping time regions, starting at $15 \mathrm{~km}$ impact parameter and extending $30 \mathrm{~km}$ upwards to $45 \mathrm{~km}$ altitude, typically corresponding to a time interval of approximately 9-10 seconds duration. (The lowest altitude for the smoothing is approximately the same lowest altitude used in the standard dual frequency correction. Below $15 \mathrm{~km}$, the ionospheric correction is extrapolated.) Successive time intervals overlap by $90 \%$. A quadratic polynomial is performed during each time interval, fitting the ionospheric estimate according to equation (3)

235 versus time, using the L1-frequency range and phase values available at 1-second cadence. Figure 2 shows a linear fit example for simplicity of notation, but quadratic fits were actually used. A comparison of linear and quadratic fitting when dual-frequency data were available for GPS/MET (108 occultations on June 23, 1995) showed that linear fitting produced a discernable bias between the single-frequency and standard dual-frequency processing, and the absence of a bias when quadratic fitting was used. A week of processing COSMIC data in January 2008 showed similar results, leading to the selection of quadratic fitting in this study. The order of polynomial fit might need to be revisited in follow-on studies using single-frequency processing nearer to solar maximum conditions.

The step to evaluate the fits is described in the bottom part of Figure 2. After fits are performed for each time interval, the final smoothed value at a given time is based on a weighted sum of the fits evaluated for each time sub-interval. The 245 weighting function is a simple linear "hat function" as diagrammed in the lower right. Weighting is maximum in the center of the sub-interval, decreasing linearly to zero at the end of each sub-interval. Weights are normalized such that the sum of all weights equals one. This weighting is chosen because the formal variance of the fit is smallest near the center of the subinterval and largest at the ends of the sub-interval. The values of the fits at the ends of the sub-intervals are given the least weight.

Examples of the ionospheric estimates based on this smoothing approach are shown in Figure 3. Plotted in each panel (a-f) are: the ionospheric estimate using phase minus range, the ionospheric estimate using dual-frequency phase (L1-L2), and the fits to these quantities. The original data are at 1 -second time intervals, but the fits are evaluated every $20 \mathrm{msec}(50 \mathrm{~Hz})$ to create ionospheric-free data for use in upstream processing because the occultation data are sampled at $50 \mathrm{~Hz}$. The altitude 255 range for the fits in 15-60 km, or whatever the upper altitude of the occultation happens to be. In Section 3, we only analyze the results up to $30 \mathrm{~km}$ altitude, but occultation data above this altitude is used in the occultation processing, so upper altitude ionospheric estimates are needed. The vertical scales in each panel deliberately differ so that detail can be seen in the variation of ionospheric delay across the occultation. The first two examples shown in Figure 3 are times when AS is on 
(encrypted L2 frequency). The remaining examples are times when AS is off, so that standard dual-frequency data are available for comparison.

Panels (a) and (b) of Figure 3 are examples where AS is on and the ionospheric estimate from the L1-L2 phase data differ strongly from the single frequency estimate. These two examples did not pass quality control (QC) using dual-frequency processing but did pass QC for single frequency processing. These examples are similar to the AS-on case presented in de la Torre et al. (2004, their Figure 1(b)) in that the variation using L1 alone is larger than using two frequencies. In addition, the dual-frequency data shows a "scalloping" behavior - linear variation followed by discontinuous jumps in phase. This may indicate that the receiver phase lock loop did not usefully track the phase of the weaker L2 frequency. Panels (c) through (f) (AS-off) show qualitatively that the two estimates of ionospheric variation are similar between dual- and single-frequency techniques. The occultations for panels (c) through (f) passed QC for single frequency processing.

In the following section, we discuss results obtained by comparing these single-frequency processed radio occultations with the dual-frequency re-processed GPS/MET dataset at CDAAC. We show evidence that averages of single-frequency profiles are not biased with respect to the dual-frequency data, suggesting that both data sets are well calibrated despite using nonconventional ionospheric processing. In the case of CDAAC, additional smoothing is applied to the $\mathrm{L} 2$ frequency to mitigate the lower SNR when AS in on. We then compare both data sets to the MERRA-2 reanalysis, which did not assimilate GPS/MET data. Comparing these three data sets suggests that reanalysis biases may exist near the tropical tropopause region during the period 1995-1997.

\subsection{Data Quantity and Characteristics}

The reprocessed CDAAC GPS/MET data set consists of 9,579 profiles spanning the period April 1995-February 1997, distributed as 5,002 with AS-off and 4,577 with AS-on. The temporal distribution of the data are shown in Figure 1. As mentioned earlier, past works that used GPS/MET data for climate or process studied tended to focus only on the two months October 1995 and February 1997. The CDAAC profiles are divided between periods of AS-on and off and so are shown separately. The JPL profiles are shown combined, since the retrieval processing does not distinguish these two conditions. The JPL single-frequency processing has yielded 4,729 profiles after QC, from an original 6,173 before a final QC was applied based on comparisons to ECMWF analysis and other factors (e.g. see Ho et al., 2009 for a description of quality control criteria). These sets are not completely overlapping: as is typical for RO from different processing centers, different criteria yield different sets of profiles that pass QC. There are 11,531 unique profiles in this combined set. This compares with less that 3,000 CDAAC AS-off profiles contained in the periods Feb 1995/Oct 1997 that were used in previously published studies. We note that JPL used both frequencies where possible for orbit determination and for calibration of the occulting link, where smoothing of the weak second frequency could be applied. Single-frequency techniques were only applied to the occultation retrieval. 
The geographic and solar local time distributions of these data sets are also shown in Figure 1. Gaps in the geographic distribution are related to the distribution of ground sites used to support GPS/MET occultations, which were undertaken when Selective Availability was in effect. Selective Availability was an intentional dithering of the GPS clock signals to reduce accuracy for receivers not specifically authorized by the US government. To remove these GPS clock errors, at least two ground sites in common view of the occulting and calibration satellites were required (Hajj et al., 2002).

\section{Results}

300 The objective of this section is to provide insight into the climate quality of GPS/MET data sets that have not received wide attention in the literature. As described below, our assessment suggests that dual-frequency processing when the L2 signal is weaker (AS-on) is not inherently less reliable than processing during the more limited periods when AS was off. We focus our analysis on the altitude range 10-30 km where the closed-loop tracking techniques implemented by the GPS/MET receiver were effective. We do not offer any conclusions about altitudes outside this range.

Clearly issues do arise during AS-on periods as evidenced by Figure 3 panels (a) and (b) (see also de la Torre et al., 2004). These appear to be cases when the receiver phase-locked-loop could not track the signal. However, such cases do not pass the subsequent QC step and are thus removed from consideration.

310 We address whether the profiles obtained during the AS-on periods are biased even though close enough to the ECMWF reanalysis to pass QC. To perform our assessment, we have compared profiles from the CDAAC data set that are common with profiles in the new JPL single-frequency data set. If modified dual-frequency processing leads to a bias with AS-on, then it is likely that these profiles will be biased with respect to the single frequency data set, since these two data sets are based on fundamentally different ways of removing ionospheric doppler shifts. The L1 frequency, which is the only

315 frequency used in the JPL approach, is not affected by AS-on or off. We discount the unlikely possibility that a bias introduced in single-frequency processing (e.g., due to multipath on the L1 range signal) will be of equal and opposite sign to the bias introduced by dual-frequency AS-on processing.

Comparisons between the CDAAC and JPL data sets, for the altitude range 10-30 km, are shown in Figure 4. This figure compares the common set of RO acquisitions that have passed QC in both data sets (2,777 profiles), which we refer to as the "profile-to-profile" (P2P) matched data set (Ho et al., 2012). These are the common set of profiles that result after independent processing and independent QC at both UCAR and JPL. Throughout the prescribed altitude range, refractivities do not deviate by more than $0.25 \%$, which roughly corresponds to temperature retrieval agreement in the range $\sim 0.5 \mathrm{~K}$ (Kuo 
et al., 2004; Appendix C). The CDAAC AS-on and AS-off retrievals are not appreciably different in comparison to the JPL retrievals. AS status is not distinguished for the JPL data set since only the L1 frequency is used in the retrievals, and L1 is not affected by AS-on or off.

The agreement between the JPL and CDAAC data sets suggests that biases in each data set are limited to at most $0.25 \%$. However, there are other possibilities. The two data sets could share a common bias that would not be revealed in this comparison. If true, this common bias would likely not be due to the presence of AS-on because the JPL processing is insensitive to that condition. Such a bias is not the subject of this work and needs to be determined by other means. Other climate-related work that has used the GPS/MET data for various purposes suggests that GPS/MET biases unrelated to AS are not significant. Another possibility is that subsets are biased in opposite signs such that the full data set is unbiased. For example, the GPS/MET retrievals (JPL or CDAAC) could have a negative bias in the southern hemisphere and a similar but opposite bias in the northern hemisphere that cancels. We will return to this subject later.

It is constructive to compare the CDAAC and JPL data sets to the MERRA-2 reanalysis, which did not assimilate GPS/MET RO data during 1995-1997. We assume that it is unlikely that biases that may exist in the RO data sets would match possible MERRA-2 biases. This comparison, shown in Figure 5, differs from Figure 4, indicating that the biases with respect to 340 MERRA-2 exceed those found between JPL and CDAAC. For example, at several altitudes the differences exceed the value of $0.25 \%$ which is a value that bounds the JPL-CDAAC comparison. Focusing on biases that exceed $0.25 \%$ in Figure 5 , we note the following features of these differences:

JPL and CDAAC AS-off versus MERRA-2 agree up to an altitude of $22 \mathrm{~km}$, where they start to diverge from each other 345 significantly.

JPL and CDAAC AS-on show a noticeable bias at $15 \mathrm{~km}$ altitude, a somewhat lower but noticeable bias at 17 and $19 \mathrm{~km}$, and no significant bias at altitudes above $23 \mathrm{~km}$ where there is a more significant bias between JPL and AS-off.

Both JPL and CDAAC AS-on have significant biases with respect to MERRA-2 starting at $23 \mathrm{~km}$ and continuing upward.

350 In summary, disagreement between the RO measurements and MERRA-2 exceed the differences between JPL and CDAAC (Figure 4), and do so in a structured way. Biases between RO and MERRA-2 increase at 15 and $17 \mathrm{~km}$, and above $22 \mathrm{~km}$.

Interpretation of Figure 5 is not straightforward. If the biases exceeding $0.25 \%$ were due solely to biases in MERRA-2, then one would not expect different biases between the three data sets plotted in Figure 5. On the other hand, if biases exceeding $3550.25 \%$ existed in the JPL or CDAAC data sets, it is difficult to explain the small differences between the JPL and CDAAC data sets (Figure 4), unless that bias were common to both JPL and CDAAC. We have already discussed why such a 
common bias is unlikely given the processing differences. At least, we could not reasonably ascribe such a common bias to AS-off processing.

One way to explain the differences shown in Figures 4 and 5 is to note that the MERRA-2 comparisons occur for different sets of profiles than the JPL-CDAAC comparisons. It is possible that different groups of profiles exhibit different biases, if the biases might be geographically or temporally dependent. We can rule out profile grouping as a factor by considering the same subset of profiles that we used in the JPL-CDAAC comparisons. Figure 6 plots the median differences with MERRA-2 for the same set of profiles used in the JPL-CDAAC comparisons, which used the common set of profiles existing in the three data sets (JPL, CDAAC AS-off and CDAAC AS-on). We label the profiles common among the three data sets as the "profile-to-profile subset" or P2P subset, following (Ho et al., 2012). We note that, although Figure 6 uses the same P2P subset of profiles used in Figure 4, comparisons between MERRA-2 and the RO profiles occur at different altitudes than comparisons between the two RO data sets (JPL and UCAR). Therefore, the differences formed for Figure 4 are not exactly the same differences as formed for Figure 6, although they involve a common set of profiles.

In Figure 5, we compared all profiles that passed QC to MERRA-2. Between the altitudes of $10 \mathrm{~km}$ and $30 \mathrm{~km}$, the biases between the retrievals and MERRA-2 show very minor differences whether all QC-passed profiles are used, or if the P2P subset is used. This suggests that the biases differences between Figures 4 and 5 are not due to the different locations of the retrievals used in these two comparisons.

\section{Discussion}

In this section, we discuss comparisons between JPL single-frequency processing, CDAAC AS-on and CDAAC AS-off dual-frequency processing, and the MERRA-2 reanalysis. Given the excellent agreement between the JPL and CDAAC data sets, and larger disagreement between these data sets and MERRA-2, we suggest that the biases exceeding $\sim 0.25 \%$ in Figure 5 are due to biases in the reanalysis, which appear to be altitude-dependent, reaching local maxima near $15 \mathrm{~km}$ and $17 \mathrm{~km}$. This conclusion is reinforced by the fact these biases are similar for different sets of profiles used in the comparisons (compare Figures 5 and 6).

That MERRA-2 biases may be more significant near 15-17 km altitudes. near the tropical tropopause, does not contradict previous studies using MERRA-2. The MERRA-2 reanalysis, despite extending from the early 1980s (Basilovich et al., 2015) does not assimilate GPS radio occultation observations until 2004 (McCarty et al., 2016). Reanalysis temperatures near the tropopause are very dependent on the vertical resolution of the model at those altitudes (Tegtmeier et al., 2020). Because absolute accuracy of the reanalysis at these altitudes, particular before RO is assimilated, is difficult to characterize, 
some studies have used comparisons between different reanalyses to gain insight. Basilovich et al. (2015) shows that comparisons between MERRA-2 and ECMWF-Interim have the largest temperature differences in the tropical tropopause region at pressure levels between about $110-130 \mathrm{hPa}$, which is near altitudes of $15-17 \mathrm{~km}$, and temperature differences approaching $1 \mathrm{~K}$ (Figure 3-5 of that work). At these altitudes, a temperature difference of $1 \mathrm{~K}$ corresponds to a refractivity difference in magnitude of approximately $0.5 \%$, which is close to the largest value observed here (Figure 5). On the other hand, a 15-year study comparing multi-mission GPS RO data to the ECMWF-Interim reanalysis finds that the refractivity differences are bounded by $0.2 \%$ in the altitude range 8-30 km (Gleisner et al., 2020). Larsen et al. (2005) report biases of up to $0.5 \mathrm{~K}$ maximizing near the tropopause for February 2000, compared to the ECMWF analyses from that period.

Biases between RO and MERRA-2 are also significant at altitudes above $20 \mathrm{~km}$, although this only occurs for the JPL and CDAAC AS-on data sets, not the CDAAC AS-off data set. Variations in bias between the data sets are also observed at 15 $\mathrm{km}$ and $17 \mathrm{~km}$. A possible reason for the bias differences between data sets is that possible biases in MERRA-2 are spatially and temporally dependent. The different locations of the profiles in the three data sets - where the comparisons are performed - could result in different biases. We view the data set differences in Figure 5, particularly the larger ones, as an opportunity to characterize the spatial/temporal bias variations that may exist in MERRA-2. Figure 5 suggests that such bias variations are altitude-dependent.

We have selected four altitudes to analyze possible latitude-dependent biases in the datasets. The altitudes are $12 \mathrm{~km}, 15$ $\mathrm{km}, 17 \mathrm{~km}$ and $26 \mathrm{~km}$ based on Figure 5. Figure 7 shows median biases between the various datasets partitioned across $15^{\circ}$ latitude bins spanning $60 \mathrm{~S}$ to $60 \mathrm{~N}$. The first three panels, which compare to MERRA-2, show the most significant bias variations with altitude occur at altitudes $15 \mathrm{~km}$ and $26 \mathrm{~km}$. Biases at $15 \mathrm{~km}$ altitude broadly peak at low latitudes, possibly corresponding to the variation of tropopause height with latitude, with higher tropopauses at the lowest latitudes. The latitudinal behavior of the biases at $15 \mathrm{~km}$ are significantly different than neighboring heights $12 \mathrm{~km}$ and $17 \mathrm{~km}$, possibly suggesting that the reanalysis in the period 1995-1997 is constrained by observations differently near the tropopause than away from it. At the higher stratospheric altitude of $26 \mathrm{~km}$, where RO becomes increasingly subject to residual biases due to ionospheric effects, all three data sets show similar latitudinal trends of more positive biases near the poles.

The bottommost panels of Figure 7 are comparisons between matched profiles between the JPL dataset and CDAAC AS-ON and OFF datasets. In both comparisons, the latitudinal dependence of the bias is much less than the MERRA-2 comparisons, regardless of altitude. This applies even to highest altitude of $26 \mathrm{~km}$, where residual ionospheric effects should be largest. The full set of panels in Figure 7 are consistent with the interpretation that the latitude dependence is due to biases in the 420 reanalysis at altitudes $17 \mathrm{~km}$ and $26 \mathrm{~km}$. The lack of RO bias at $26 \mathrm{~km}$ is perhaps not surprising, despite the different processing, because of the solar minimum conditions during 1995-1997 when these data were acquired. 


\subsection{Wider application of single-frequency processing}

Single-frequency processing was developed for this paper to provide insight on the robustness of the complete GPS/MET datasets from 1995-1997, as published at the CDAAC site. These data are now augmented by a single-frequency processed data set available from JPL's GENESIS site. In this section, we discuss other applications where single frequency processing may be useful.

In the early days of RO, dual-frequency processing was more challenging because so-called codeless processing was not yet implemented that increases L2 SNR even in the presence of Anti-Spoofing encryption (Kursinski et al., 1996). Single frequency processing has been applied to the Oersted RO dataset for this reason (Larsen et al., 2005). Even now, the second GNSS frequency is only tracked at altitudes above 10-20 km because the L2 frequency is weaker due to encryption and often too weak to track at lower altitudes. Instead, extrapolation of the dual-frequency correction computed at higher altitudes is performed below this cutoff, which can incur ionospheric-dependent biases when the extrapolation is not accurate due to ionospheric structure. At these lower altitudes, the effect of residual ionosphere is decreasing (Mannucci et al., 2006) but there may be instances near solar maximum where further analysis of this extrapolation error is warranted.

The second-generation GRAS receivers planned for MetOp do not track the legacy encrypted L2 signals, since there are sufficient numbers of satellites transmitting the newer unencrypted dual-frequency signals. However, single frequency techniques could be used on the remaining satellites from the GPS system that do not yet transmit the newer signals, of which there may still be some for several more years.

Another application where single-frequency techniques may be valuable is in investigations of residual systematic effects of the standard dual-frequency correction. This residual is primarily due to raypath separation between the L1 and L2 signal paths, sampling different parts of the ionosphere (Vorob'ev and Krasil'nikova, 1994; Syndergaard, 2000). The reason such a residual cannot be removed easily is because the electron density gradients in the ionosphere are not known with sufficient accuracy, thus the different paths in the ionosphere are not known. Comparing the single and dual-frequency correction methods may provide some information on the magnitude of the residual due to this raypath separation to augment simulation studies of this error source (Danzer et al., 2013; Danzer et al. 2020). Danzer et al. (2013) estimate that fractional refractivity errors due to raypath separation in the ionosphere can reach $\sim 1 \%$ near $30 \mathrm{~km}$ altitude for solar maximum conditions. The analysis in this paper suggests that single-frequency retrieval error is less than $0.25 \%$ for solar activity conditions closer to minimum (1995-1997). Single-frequency retrieval errors may not depend strongly on solar cycle since they depend primarily on pseudorange noise (random and systematic due to multipath). Therefore, the single frequency technique could provide information on the residual ionospheric bias affecting ensembles of dual-frequency daytime profiles despite that single frequency processing exhibits higher random error per profile because of the pseudorange noise. 
Healy and Culverwell (2015) proposed so the so-called "Kappa" technique based on a formula in the Vorob'ev and Krasil'nikova (1994) paper that discussed applying the dual-frequency correction in bending angle space (Healy and Culverwell, 2015). Evaluation of this correction has been promising (Danzer et al., 2015; Danzer et al., 2020) although we note that its efficacy will vary with satellite altitude. For example, as shown in the study by Mannucci et al. (2011), there is an error cancellation effect for satellite altitudes well above the height of peak ionospheric vertical gradients, which typically occur just below the altitude of peak density. The occulting ray first enters the ionosphere from above after leaving the GNSS transmitter. Then the signal exits the ionosphere and re-enters from below as it propagates to the LEO. If the LEO is orbiting in the ionospheric topside (well above the F-layer density peak), then the residual error approximately cancels between the entrance and exit phases, although not fully because of ionospheric differences between entry and exit regions. Additional insights are available in Danzer et al. (2020) and Li et al. (2020).

Residual ionospheric errors due to the path separation effect is much reduced for the single-frequency correction method, because the bandwidth required to construct the group delay signal at L1 is much less than the frequency separation between L1 and L2 (10 MHz for L1 versus $348 \mathrm{MHz}$ for the L1/L2 combination). Thus, residual systematic errors resulting from the single frequency technique (ignoring errors due to multipath on the L1 ranging code) will differ significantly from the L1/L2 technique. (We note that this argument does not apply to residuals from higher-order terms in the Appleton-Hartree expansion, which affects the $\mathrm{CA} / \mathrm{L} 1$ approximation roughly similarly to the $\mathrm{L} 1 / \mathrm{L} 2$ approximation).

\section{Conclusions}

475 We have performed an analysis of reprocessed GPS/MET data spanning 1995-1997 generated by CDAAC in 2007. CDAAC developed modified dual-frequency processing methods for the encrypted data (AS-on) during 1995-1997. We compared the CDAAC data set to the MERRA-2 reanalysis, separately for AS-on and AS-off, focusing on the altitude range 10-30 km. MERRA-2 did not assimilate GPS/MET data in the period 1995-1997. To gain insight into the CDAAC data set, we developed a single-frequency data set for GPS/MET, which is unaffected by the presence of encryption. We find excellent agreement between the more limited single frequency data set and the CDAAC data set: the bias between these two data sets is consistently less than $0.25 \%$ in refractivity, whether AS is on or not. Given the different techniques applied between the CDAAC and JPL data sets, agreement suggests that the CDAAC AS-on processing and the single frequency processing are not biased in an aggregate sense greater than $0.25 \%$ in refractivity, which corresponds approximately to a temperature bias less than $0.5 \mathrm{~K}$.

Since the profiles contained in the new single frequency data set are not a subset of the CDAAC profiles, the combination of the CDAAC data set, consisting of 9,579 profiles, and the new single-frequency data set, consisting of 4,729 profiles, yields 
a total number of 11,531 unique profiles from combining the JPL and CDAAC data sets. All numbers refer to quality control having been applied.

We performed comparisons between the GPS/MET data sets and the MERRA-2 reanalysis. The biases between the observations and MERRA-2 exceeded the biases between the JPL and CDAAC data sets, suggesting possible altitudedependent biases in MERRA-2. The CDAAC AS-off data set generally had the smallest bias with respect to MERRA-2, although increased biases appeared at $15 \mathrm{~km}$ altitude and near $30 \mathrm{~km}$ altitude. The bias between MERRA-2 and the CDAAC AS-on data set reached a local peak near $17 \mathrm{~km}$ altitude, starting to increase above $18 \mathrm{~km}$ altitude, eventually reaching nearly $0.75 \%$ difference. Similarly, the JPL data set bias increased above $18 \mathrm{~km}$ altitude, and exhibited a local peak near $15 \mathrm{~km}$.

Considering that the differences between MERRA-2 and the three data sets (JPL, CDAAC AS-off and CDAAC AS-on) are not identical, it is possible that the different times and locations of the three data sets could be responsible for the varying differences with MERRA-2. Differences between observations and MERRA-2 were also computed for a limited set of occultation events that were common between JPL and CDAAC AS-on, and between JPL and CDAAC AS-off. The biases between observations and MERRA-2 were similar whether a common set of profiles were used or not. This suggests that the locations of the radio location profiles were not a significant determining factor in the bias differences between the retrievals and MERRA-2.

We examined the biases between the retrievals and MERRA-2 as a function of latitude band and altitude. At $15 \mathrm{~km}$ altitude, the biases between all three retrieval sets and MERRA-2 have a broad peak near low latitudes. The opposite trend is seen at $26 \mathrm{~km}$ altitude: the biases are largest at the high latitudes (north and south). At $17 \mathrm{~km}$, only the JPL data set shows significant variation with latitude. In contrast, there is less variation of bias with latitude between JPL and the two CDAAC data sets (AS-on, AS-off).

Single frequency processing of RO data can be a useful method to compare with dual-frequency methods that are subject to a residual ionospheric error (RIE). Although other errors can affect single frequency processing, such as code multipath, these errors are likely independent of the ionospheric residual. Therefore, ensembles of profiles using single frequency processing could be used as an independent check on RIE after being validated against dual-frequency profiles during periods when the RIE is negligible, e.g. at nighttime or during solar minimum. Single frequency processing holds promise when two frequencies are unavailable or as a means of validating L2 processing when a new receiver is being evaluated that uses special L2 processing to overcome encryption. Two previous studies discuss the potential value of single frequency processing (Larsen et al., 2005; de la Torre Juarez et al., 2004). 
Based on these results, future reanalyses should consider using the full combined data set of single- and dual-frequency processed data from GPS/MET, consisting of 11,531 unique profiles. Healy et al. (2017) found positive impact on the ERA5 reanalysis after assimilating UCAR GPS/MET data from a limited AS-on period spanning December 23, 1996 to January 13, 1997.

\section{Data Availability}

The UCAR CDAAC data set can be found at: http://cdaac-www.cosmic.ucar.edu. The new single-frequency data set can be found at: TBD on paper acceptance.

\section{Author Contribution}

A. J. Mannucci led the conceptualization, investigation and methodology, as well as contributing to validation and analysis software. C. Ao contributed to the methodology, validation and software. B. Iijima contributed to the investigation and software used in this work. T. Meehan contributed ideas to the investigation. P. Vergados contributed to the investigation and provided useful review and editing of the manuscript. E. R. Kursinski contributed to the methodology, validation, and provided review and editing. S. Healy and A. Horányi provided review and editing of the manuscript and elements of the conceptualization and methodology. W. S. Schreiner provided inputs on the methodology and validation, and review and editing.

\section{Competing Interests}

540 The authors declare that they have no conflict of interest.

\section{Acknowledgements}

Portions of this research were carried out at the Jet Propulsion Laboratory, California Institute of Technology, under a contract with the National Aeronautics and Space Administration. Support of the NASA Earth science division is 545 acknowledged. 
https://doi.org/10.5194/amt-2021-241

Preprint. Discussion started: 25 August 2021

(C) Author(s) 2021. CC BY 4.0 License.
Atmospheric

Measurement

Techniques

Discussions

\section{References}

Anthes, R., and Rieckh, T.: Estimating observation and model error variances using multiple data sets, Atmospheric Measurement Techniques, 11, 4239-4260, 10.5194/amt-11-4239-2018, 2018.

Bosilovich, M. G., Akella, S., Coy, L., Cullather, R., and Draper, C.: MERRA-2: Initial evaluation of the climate, 2015.

Danzer, J., Healy, S. B., and Culverwell, I. D.: A simulation study with a new residual ionospheric error model for GPS radio occultation climatologies, Atmospheric Measurement Techniques, 8, 3395-3404, 10.5194/amt-8-3395-2015, 2015.

Danzer, J., Scherllin-Pirscher, B., and Foelsche, U.: Systematic residual ionospheric errors in radio occultation data and a potential way to minimize them, Atmospheric Measurement Techniques, 6, 2169-2179, 10.5194/amt-6-2169-2013, 2013.

Danzer, J., Schwaerz, M., Kirchengast, G., and Healy, S. B.: Sensitivity Analysis and Impact of the Kappa-Correction of Residual Ionospheric Biases on Radio Occultation Climatologies, Earth and Space Science, 7, D24110-24116, 10.1029/2019EA000942, 2020.

Davies, K.: Ionospheric Radio, 1 ed., The Institution of Engineering and Technology, Stevenage, United Kingdom, 1990.

de la Torre Juárez, M., Hajj, G. A., Kursinski, E. R., Kuang, D., Mannucci, A. J., and Romans, L. J.: Single frequency processing of atmospheric radio occultations, International Journal of Remote Sensing, 25, 3731-3744, 10.1080/0143116031000156800, 2004.

GCOS, The global observing system for climate: implementation needs. GCOS-200, Global Climate Observing System, World Meteorological Organization, 316 pp., 2016.

Gleisner, H., Lauritsen, K. B., Nielsen, J. K., and Syndergaard, S.: Evaluation of the 15-year ROM SAF monthly mean GPS radio occultation climate data record, Atmospheric Measurement Techniques, 13, 3081-3098, 10.5194/amt-13-3081-2020, 2020.

Gorbunov, M. E., and Kornblueh, L.: Analysis and validation of GPS/MET radio occultation data, Journal of Geophysical ResearchAtmospheres, 106, 17161-17169, 10.1029/2000JD900816, 2001.

Hajj, G. A., Kursinski, E. R., Romans, L. J., and Bertiger, W. I.: A technical description of atmospheric sounding by GPS occultation, Journal of Atmospheric and Solar-Terrestrial Physics, 64, 451-469, 10.1016/s1364-6826(01)00114-6, 2002.

Healy, S. B., and Culverwell, I. D.: A modification to the standard ionospheric correction method used in GPS radio occultation, Atmospheric Measurement Techniques, 8, 3385-3393, 10.5194/amt-8-3385-2015, 2015.

Healy, S.B., A. Horányi and A. Simmons (2017) Assessing the impact of GPS radio occultation measurements in ERA5, poster presenter the 5th International Conference on Reanalysis, Rome, Italy, 13-17 November. https://www.romsaf.org/Publications/conferences/Healy rome v1 SW.pdf

Ho, S.-P., Kirchengast, G., Leroy, S., Wickert, J., Mannucci, A. J., Steiner, A., Hunt, D., Schreiner, W., Sokolovskiy, S., Ao, C., Borsche, M., von Engeln, A., Foelsche, U., Heise, S., Iijima, B., Kuo, Y.-H., Kursinski, R., Pirscher, B., Ringer, M., Rocken, C., and Schmidt, T.: Estimating the uncertainty of using GPS radio occultation data for climate monitoring: Intercomparison of CHAMP refractivity climate records from 2002 to 2006 from different data centers, Journal of Geophysical Research: Atmospheres, 114, D23107, 10.1029/2009JD011969, 2009.

Ho, S.-P., Hunt, D., Steiner, A. K., Mannucci, A. J., Kirchengast, G., Gleisner, H., Heise, S., Engeln, A., Marquardt, C., Sokolovskiy, S., Schreiner, W., Scherllin-Pirscher, B., Ao, C., Wickert, J., Syndergaard, S., Lauritsen, K. B., Leroy, S., Kursinski, E. R., Kuo, Y.-H., Foelsche, U., Schmidt, T., and Gorbunov, M.: Reproducibility of GPS radio occultation data for climate monitoring: Profile-to-profile inter-comparison of CHAMP climate records 2002 to 2008 from six data centers, Journal of Geophysical Research: Atmospheres, 117, D18111, 10.1029/2012JD017665, 2012.

Kuo, Y. H., Wee, T. K., Sokolovskiy, S., Rocken, C., Schreiner, W., Hunt, D., and Anthes, R. A.: Inversion and Error Estimation of GPS Radio Occultation Data, Journal of the Meteorological Society of Japan. Ser. II, 82, 507-531, 10.2151/jmsj.2004.507, 2004.

Kursinski, E. R., Hajj, G. A., Bertiger, W. I., Leroy, S. S., Meehan, T. K., Romans, L. J., Schofield, J. T., McCleese, D. J., Melbourne, W. G., Thornton, C. L., Yunck, T. P., Eyre, J. R., and Nagatani, R. N.: Initial Results of Radio Occultation Observations of Earth\&apos;s Atmosphere Using the Global Positioning System, Science, 271, 1107-1110, 10.1126/science.271.5252.1107, 1996.

Kursinski, E. R., Hajj, G. A., Schofield, J. T., Linfield, R. P., and Hardy, K. R.: Observing Earth\&amp;apos;s atmosphere with radio occultation measurements using the Global Positioning System, Journal of Geophysical Research: Atmospheres, 102, 2342923465, 10.1029/97JD01569, 1997.

Lackner, B. C., Steiner, A. K., Hegerl, G. C., and Kirchengast, G.: Atmospheric Climate Change Detection by Radio Occultation Data Using a Fingerprinting Method, Journal of Climate, 24, 5275-5291, 10.1175/2011JCLI3966.1, 2011. 
https://doi.org/10.5194/amt-2021-241

Preprint. Discussion started: 25 August 2021

(C) Author(s) 2021. CC BY 4.0 License.
Atmospheric

Measurement

Techniques

Discussions

Larsen, G. B., Syndergaard, S., Høeg, P., and Sørensen, M. B.: Single frequency processing of Ørsted GPS radio occultation measurements, GPS Solutions, 9, 144-155, 10.1007/s10291-005-0142-x, 2005.

Leroy, S. S., Anderson, J. G., and Dykema, J. A.: Testing climate models using GPS radio occultation: A sensitivity analysis, Journal of Geophysical Research, 111, D17105, 10.1029/2005JD006145, 2006.

Leroy, S. S., Anderson, J. G., and Ohring, G.: Climate Signal Detection Times and Constraints on Climate Benchmark Accuracy Requirements, Journal of Climate, 21, 841-846, 10.1175/2007JCLI1946.1, 2008.

Li, M., Yue, X., Wan, W., and Schreiner, W. S.: Characterizing Ionospheric Effect on GNSS Radio Occultation Atmospheric Bending Angle, Journal of geophysical research, 125, 507-515, 10.1029/2019JA027471, 2020.

Mannucci, A. J., Ao, C. O., Pi, X., and Iijima, B. A.: The impact of large scale ionospheric structure on radio occultation retrievals, Atmospheric Measurement Techniques, 4, 2837-2850, 10.5194/amt-4-2837-2011, 2011.

Mannucci, A. J., Ao, C. O., Yunck, T. P., Young, L. E., Hajj, G. A., Iijima, B. A., Kuang, D., Meehan, T. K., and Leroy, S. S.: Generating climate benchmark atmospheric soundings using GPS occultation data, SPIE Optics + Photonics, Sep 01, 2006.

Marquardt, C., Labitzke, K., Reigber, C., Schmidt, T., and Wickert, J.: An assessment of the quality of GPS/MET radio limb soundings during February 1997, Physics and Chemistry of the Earth Part a-Solid Earth and Geodesy, 26, 125-130, 10.1016/S1464-1895(01)00035-7, 2001.

McCarty, W., Coy, L., Gelaro, R., Huang, A., Merkova, D., Smith, E. B., Sienkiewicz, M., and Wargan, K.: MERRA-2 Input Observations: Summary and Assessment, 1-64, 2016.

Nishida, M., Shimizu, A., Tsuda, T., Rocken, C., and Ware, R. H.: Seasonal and Longitudinal Variations in the Tropical Tropopause Observed with the GPS Occultation Technique (GPS/MET), Journal of the Meteorological Society of Japan. Ser. II, 78, 691-700, 10.2151/jmsj1965.78.6_691, 2000.

Poli, P., Healy, S. B., and Dee, D. P.: Assimilation of Global Positioning System radio occultation data in the ECMWF ERA-Interim reanalysis, Quarterly Journal of the Royal Meteorological Society, 136, 1972-1990, 10.1002/qj.722, 2010.

Randel, W. J., F., W., and W., R. R.: Thermal variability of the tropical tropopause region derived from GPS/MET observations, Journal of Geophysical Research, 108, 1394-1312, 10.1029/2002JD002595, 2003.

Rocken, C., Anthes, R., Exner, M., Hunt, D., Sokolovskiy, S., Ware, R., Gorbunov, M., Schreiner, W., Feng, D., Herman, B., Kuo, Y. H., and Zou, X.: Analysis and validation of GPS/MET data in the neutral atmosphere, Journal of Geophysical Research: Oceans, 102, 29849-29866, 10.1029/97JD02400, 1997.

Ruston, B., and Healy, S.: Forecast Impact of FORMOSAT-7/COSMIC-2 GNSS Radio Occultation Measurements, Atmospheric Science Letters, 36, L17809, 10.1002/asl.1019, 2020.

Santer, B. D., Solomon, S., Wentz, F. J., Fu, Q., Po-Chedley, S., Mears, C., Painter, J. F., and Bonfils, C.: Tropospheric Warming Over The Past Two Decades, Sci Rep, 7, 2336, 10.1038/s41598-017-02520-7, 2017.

Schreiner, W., Hunt, D., and Rocken, C.: Precise GPS data processing for the GPS/MET radio occultation mission at UCAR, PROCEEDINGS OF THE ..., 3389476015724488147related:0-X2Y3rWCS8J, 1998.

Steiner, A. K., Hunt, D., Ho, S. P., Kirchengast, G., Mannucci, A. J., Scherllin-Pirscher, B., Gleisner, H., von Engeln, A., Schmidt, T., Ao, C., Leroy, S. S., Kursinski, E. R., Foelsche, U., Gorbunov, M., Heise, S., Kuo, Y. H., Lauritsen, K. B., Marquardt, C., Rocken, C., Schreiner, W., Sokolovskiy, S., Syndergaard, S., and Wickert, J.: Quantification of structural uncertainty in climate data records from GPS radio occultation, Atmospheric Chemistry and Physics, 13, 1469-1484, 10.5194/acp-13-1469-2013, 2013.

Steiner, A. K., and Kirchengast, G.: Error analysis for GNSS radio occultation data based on ensembles of profiles from end-to-end simulations, Journal of Geophysical Research: Atmospheres, 110, D15307, 10.1029/2004JD005251, 2005.

Steiner, A. K., Kirchengast, G., Lackner, B. C., Pirscher, B., Borsche, M., and Foelsche, U.: Atmospheric temperature change detection with GPS radio occultation 1995 to 2008, Geophysical Research Letters, 36, L18702-18705, 10.1029/2009GL039777,

6352009.

Steiner, A. K., Lackner, B. C., Ladstädter, F., Scherllin-Pirscher, B., Foelsche, U., and Kirchengast, G.: GPS radio occultation for climate monitoring and change detection, Radio Science, 46, n/a-n/a, 10.1029/2010RS004614, 2011.

Steiner, A. K., Ladstadter, F., Ao, C. O., Gleisner, H., Ho, S. P., Hunt, D., Schmidt, T., Foelsche, U., Kirchengast, G., Kuo, Y. H., Lauritsen, K. B., Mannucci, A. J., Nielsen, J. K., Schreiner, W., Schwarz, M., Sokolovskiy, S., Syndergaard, S., and Wickert, J.: Consistency and structural uncertainty of multi-mission GPS radio occultation records, Atmospheric Measurement Techniques, 13, 2547-2575, 10.5194/amt-13-2547-2020, 2020.

Steiner, A. K., Ladstädter, F., Randel, W. J., Maycock, A. C., Fu, Q., Claud, C., Gleisner, H., Haimberger, L., Ho, S. P., Keckhut, P., Leblanc, T., Mears, C., Polvani, L. M., Santer, B. D., Schmidt, T., Sofieva, V., Wing, R., and Zou, C. Z.: Observed Temperature 
https://doi.org/10.5194/amt-2021-241

Preprint. Discussion started: 25 August 2021

(C) Author(s) 2021. CC BY 4.0 License.

\section{Atmospheric \\ Measurement \\ Techniques \\ Discussions}

645

Changes in the Troposphere and Stratosphere from 1979 to 2018, Journal of Climate, 33, 8165-8194, 10.1175/JCLI-D-19-0998.1, 2020.

Syndergaard, S.: On the ionosphere calibration in GPS radio occultation measurements, Radio Science, 35, 865-883, 10.1029/1999RS002199, 2000.

Tegtmeier, S., Anstey, J., Davis, S., Dragani, R., Harada, Y., Ivanciu, I., Pilch Kedzierski, R., Krüger, K., Legras, B., Long, C., Wang, J. S., Wargan, K., and Wright, J. S.: Temperature and tropopause characteristics from reanalyses data in the tropical tropopause layer, Atmospheric Chemistry and Physics, 20, 753-770, 10.5194/acp-20-753-2020, 2020.

Vergados, P., and Pagiatakis, S. D.: Latitudinal, solar, and vertical variability of higher-order ionospheric effects on atmospheric parameter retrievals from radio occultation measurements, Journal of Geophysical Research: Space Physics, 116, https://doi.org/10.1029/2011JA016573, 2011.

Verkhoglyadova, O. P., Mannucci, A. J., Ao, C. O., Iijima, B. A., and Kursinski, E. R.: Effect of small-scale ionospheric variability on GNSS radio occultation data quality, Journal of geophysical research, 120, 7937-7951, 10.1002/2015JA021055, 2015.

von Engeln, A., Healy, S., Marquardt, C., Andres, Y., and Sancho, F.: Validation of operational GRAS radio occultation data, Geophysical ..., 36, L17809, 10.1029/2009GL039968, 2009.

Vorobev, V. V., and KrasIlnIkova, T. G.: Estimation of the accuracy of the atmospheric refractive index recovery from Doppler shift measurements at frequencies used in the NAVSTAR system, Izvestiya Academy of Sciences SSSR, English Translation, Atmospheric and Oceanic Physics, 29, 602-609, 1994.

Wielicki, B. A., Young, D. F., Mlynczak, M. G., Thome, K. J., Leroy, S., Corliss, J., Anderson, J. G., Ao, C. O., Bantges, R., Best, F., Bowman, K., Brindley, H., Butler, J. J., Collins, W., Dykema, J. A., Doelling, D. R., Feldman, D. R., Fox, N., Huang, X., Holz, R., Huang, Y., Jin, Z., Jennings, D., Johnson, D. G., Jucks, K., Kato, S., Kirk-Davidoff, D. B., Knuteson, R., Kopp, G., Kratz, D. P., Liu, X., Lukashin, C., Mannucci, A. J., Phojanamongkolkij, N., Pilewskie, P., Ramaswamy, V., Revercomb, H., Rice, J., C., Tobin, D., and Xiong, X.: Achieving Climate Change Absolute Accuracy in Orbit, Bulletin of the American Meteorological Society, 94, 1519-1539, 10.1175/BAMS-D-12-00149.1, 2013. 
https://doi.org/10.5194/amt-2021-241

Preprint. Discussion started: 25 August 2021

(C) Author(s) 2021. CC BY 4.0 License.

(c) (i)
Atmospheric Measurement Techniques

Discussions

670
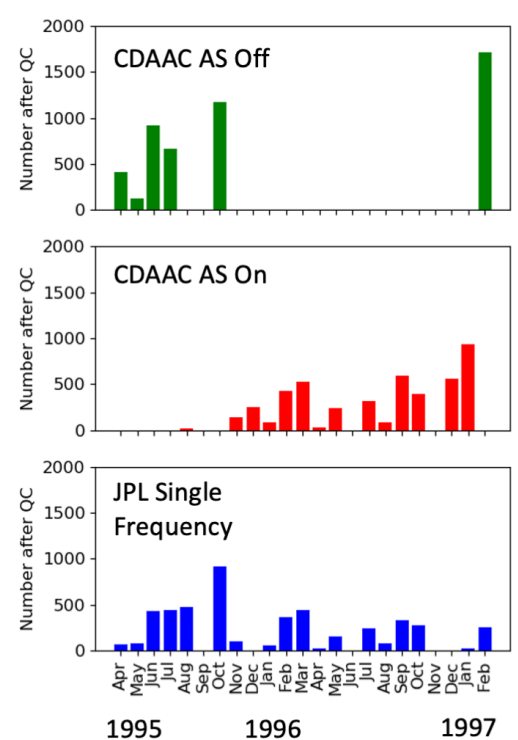
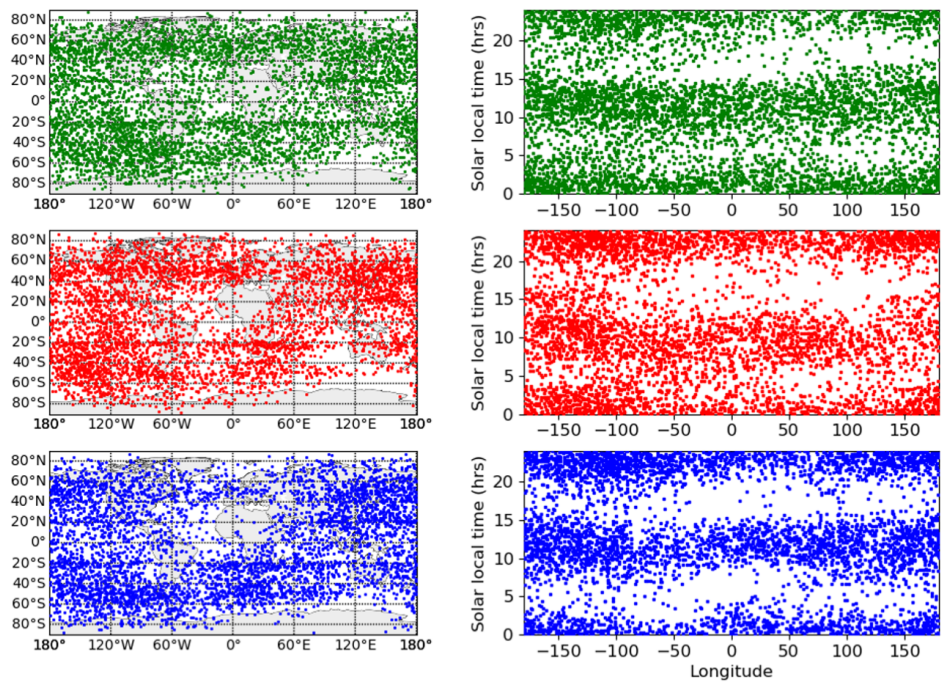

Figure 1. Information on the three data sets analyzed: UCAR's CDAAC AS-off, AS-on and JPL single frequency. The left column shows the number of profiles by month, after quality control is applied. The middle column shows the locations of the profiles. The right column shows the solar local time of the profiles versus longitude. 


\section{Fitting step:}

1. Define overlapping time intervals $(90 \%)$

2. Fit polynomial over each interval

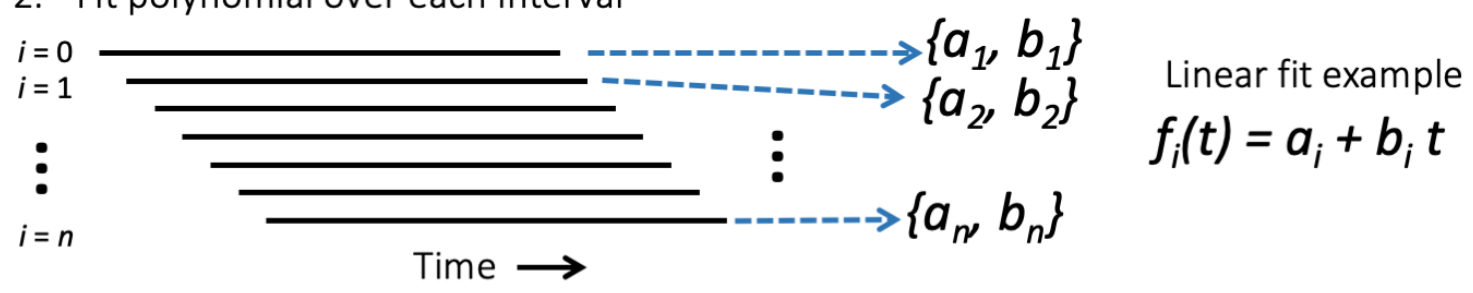

Evaluation step:

1. Evaluate each fit $i$ at time $t$

2. Perform a weighted sum over each evaluation

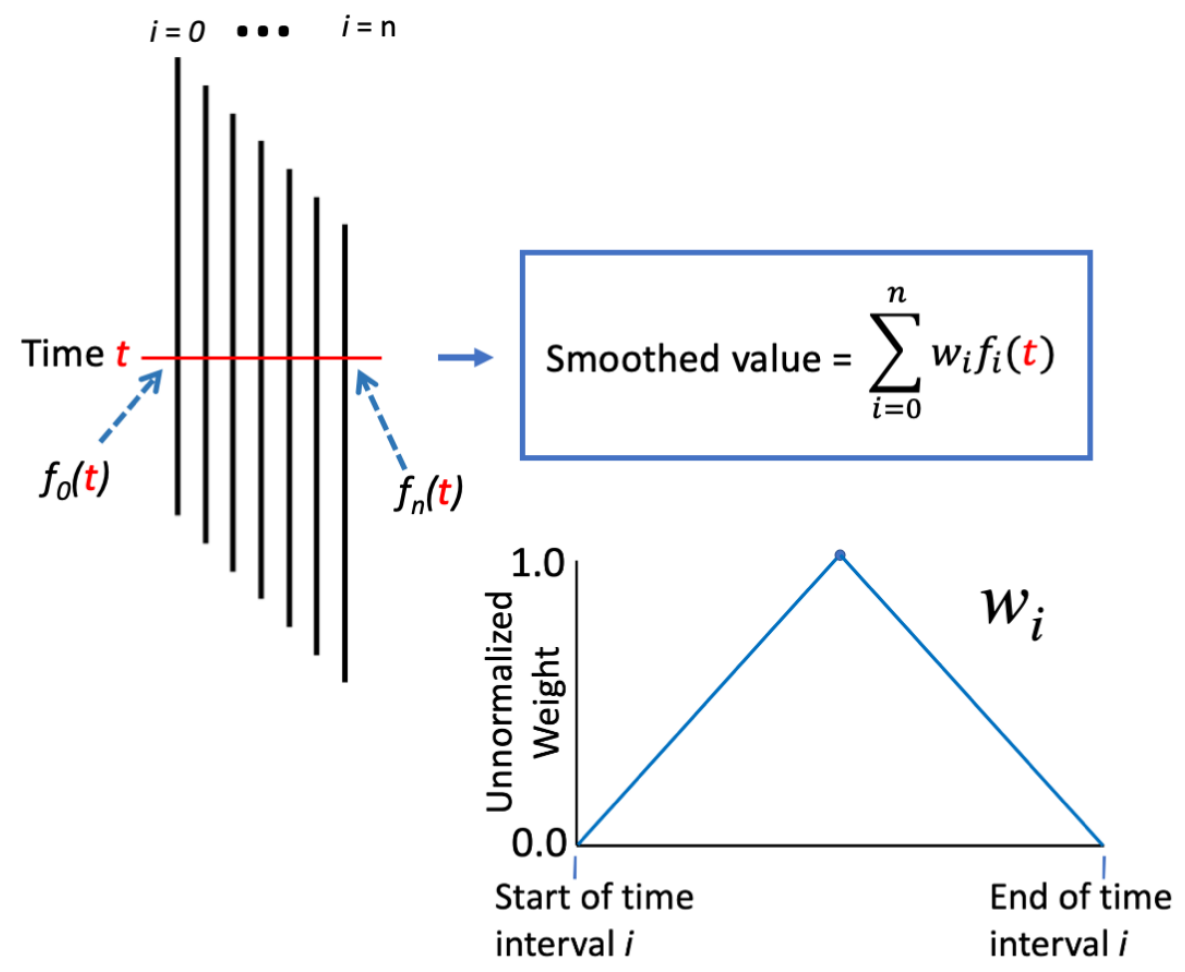

Figure 2. Illustration of the smoothing process used in single-frequency processing. This example shows linear fits to the segments, although quadratic fits were actually used. 
https://doi.org/10.5194/amt-2021-241

Preprint. Discussion started: 25 August 2021

(c) Author(s) 2021. CC BY 4.0 License.
Atmospheric Measurement

Techniques

Discussions
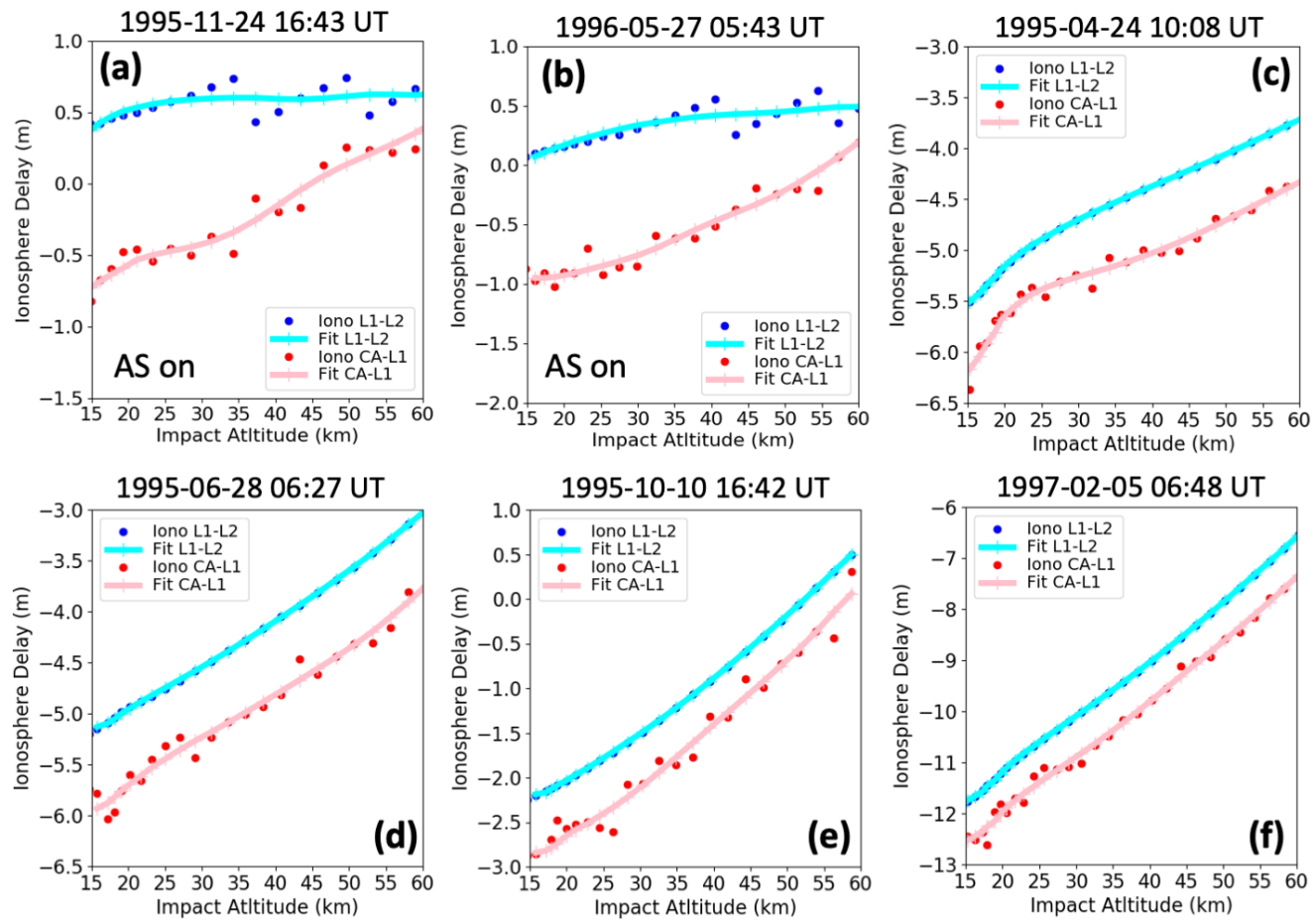

Figure 3. Examples of ionospheric delays using the single-frequency method and the smoothing approach illustrated in Figure 2 (pink). Panels (a) and (b) are examples where AS is on, and the L2 frequency is much weaker. Panels (c)(f) correspond to AS-off, and standard dual-frequency techniques could be used. Ionospheric delays from dualfrequency processing are shown for comparison (blue). 


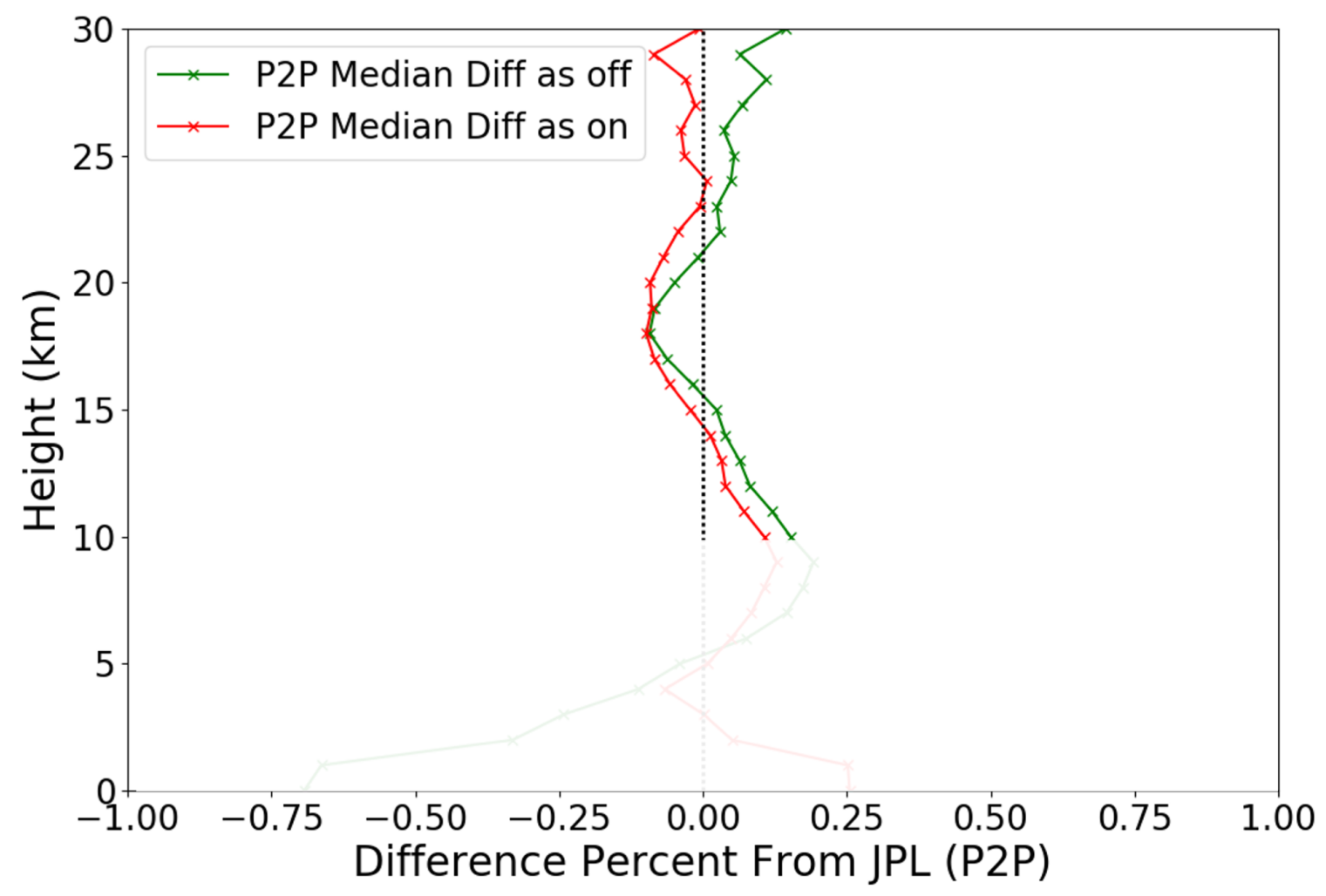

690 Figure 4. Percentage refractivity median difference of JPL minus UCAR AS-on and AS-off for profiles common to the single frequency and UCAR data sets ("P2P" data set). The focus altitudes are $10 \mathrm{~km}-30 \mathrm{~km}$. Profiles are interpolated to a common 1-km vertical grid spacing. 


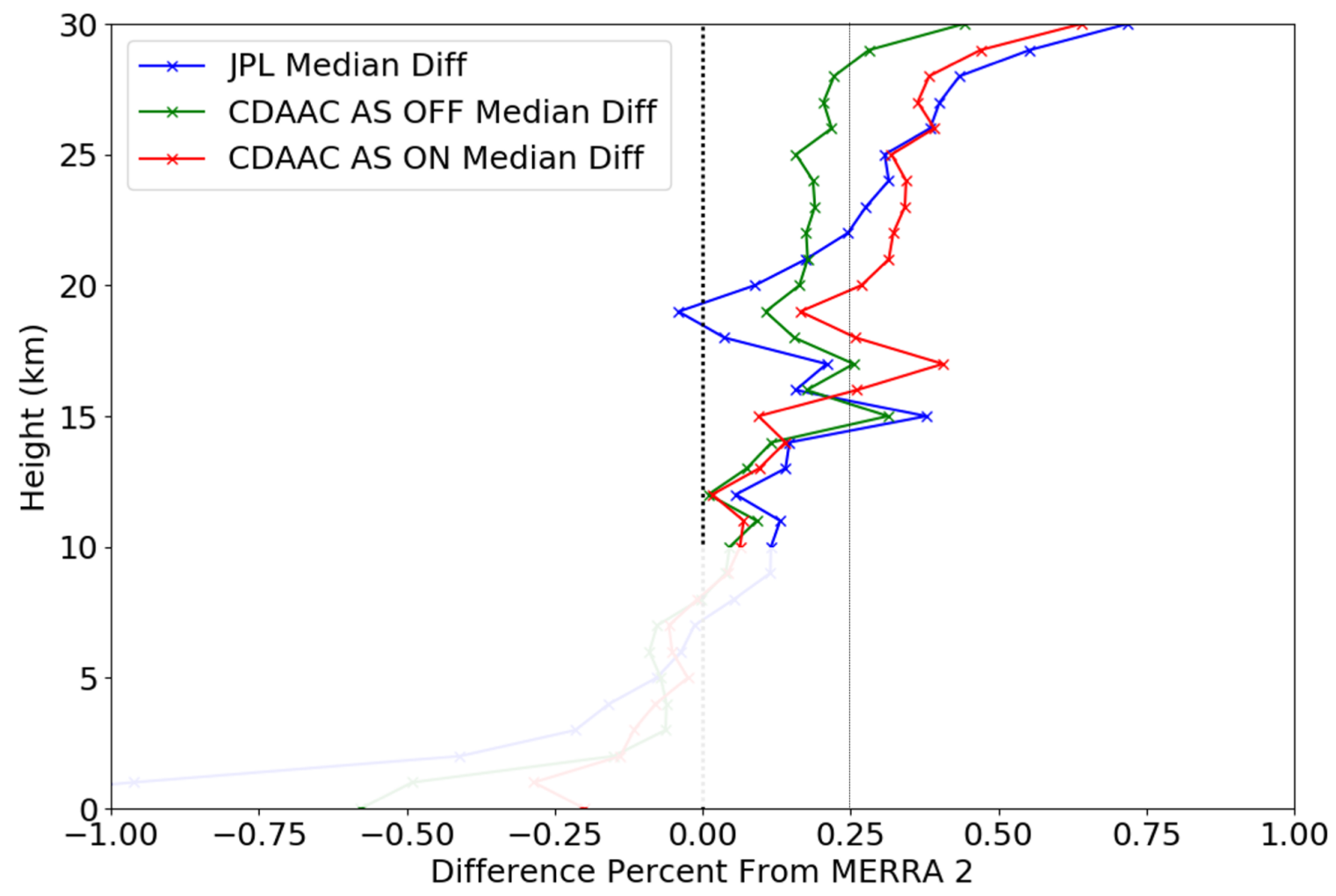

Figure 5. Percentage refractivity median differences of the JPL and CDAAC data sets minus the MERRA-2 reanalysis, interpolated to a common 1-km altitude grid. Altitudes between 10 and $30 \mathrm{~km}$ are analyzed. 


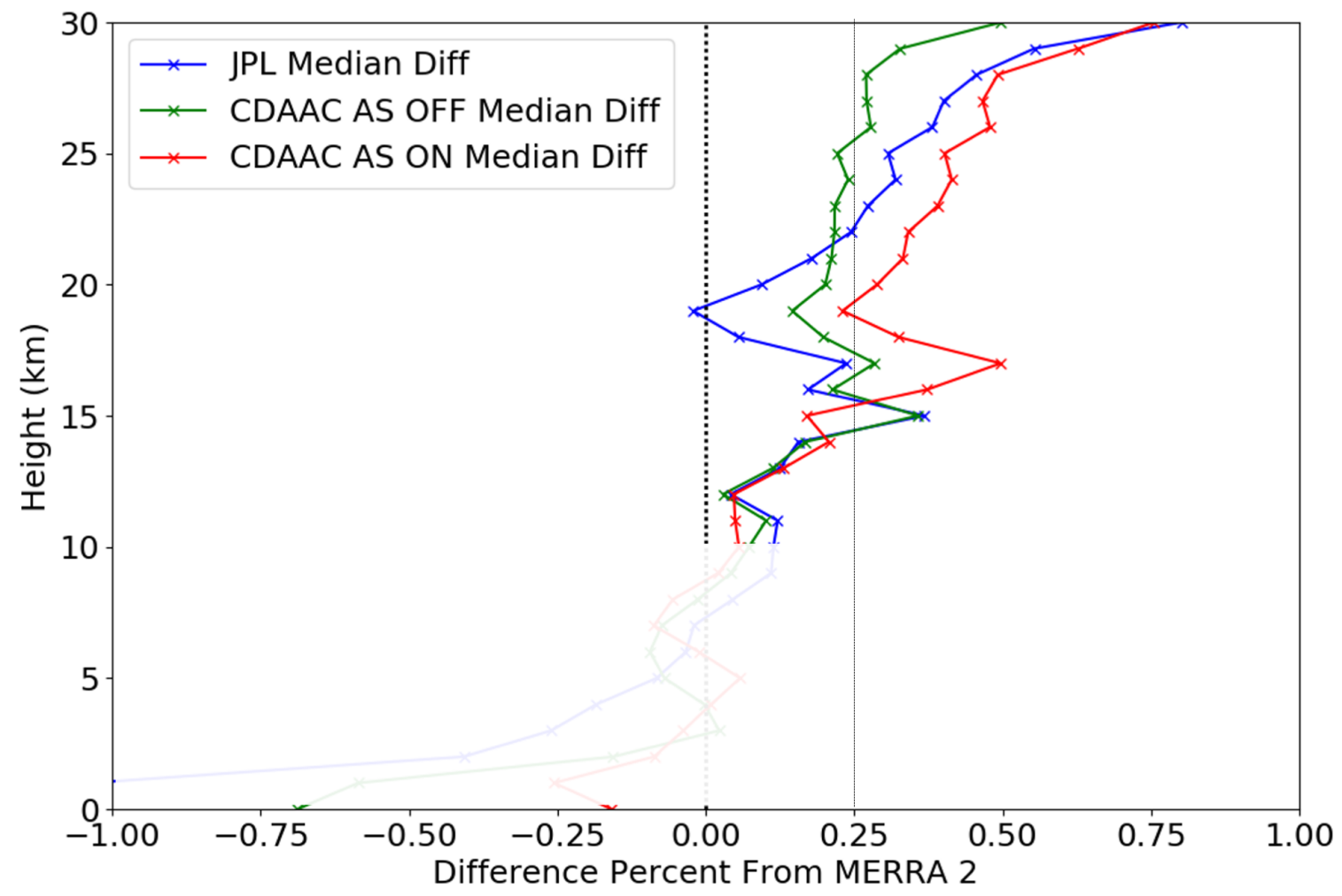

Figure 6. Similar to Figure 5 except using only the set of profiles common between the JPL and CDAAC data sets that is the same set used in the comparison shown in Figure 4. 
https://doi.org/10.5194/amt-2021-241

Preprint. Discussion started: 25 August 2021

(c) Author(s) 2021. CC BY 4.0 License.

(c) (i)
Atmospheric Measurement

Techniques

Discussions
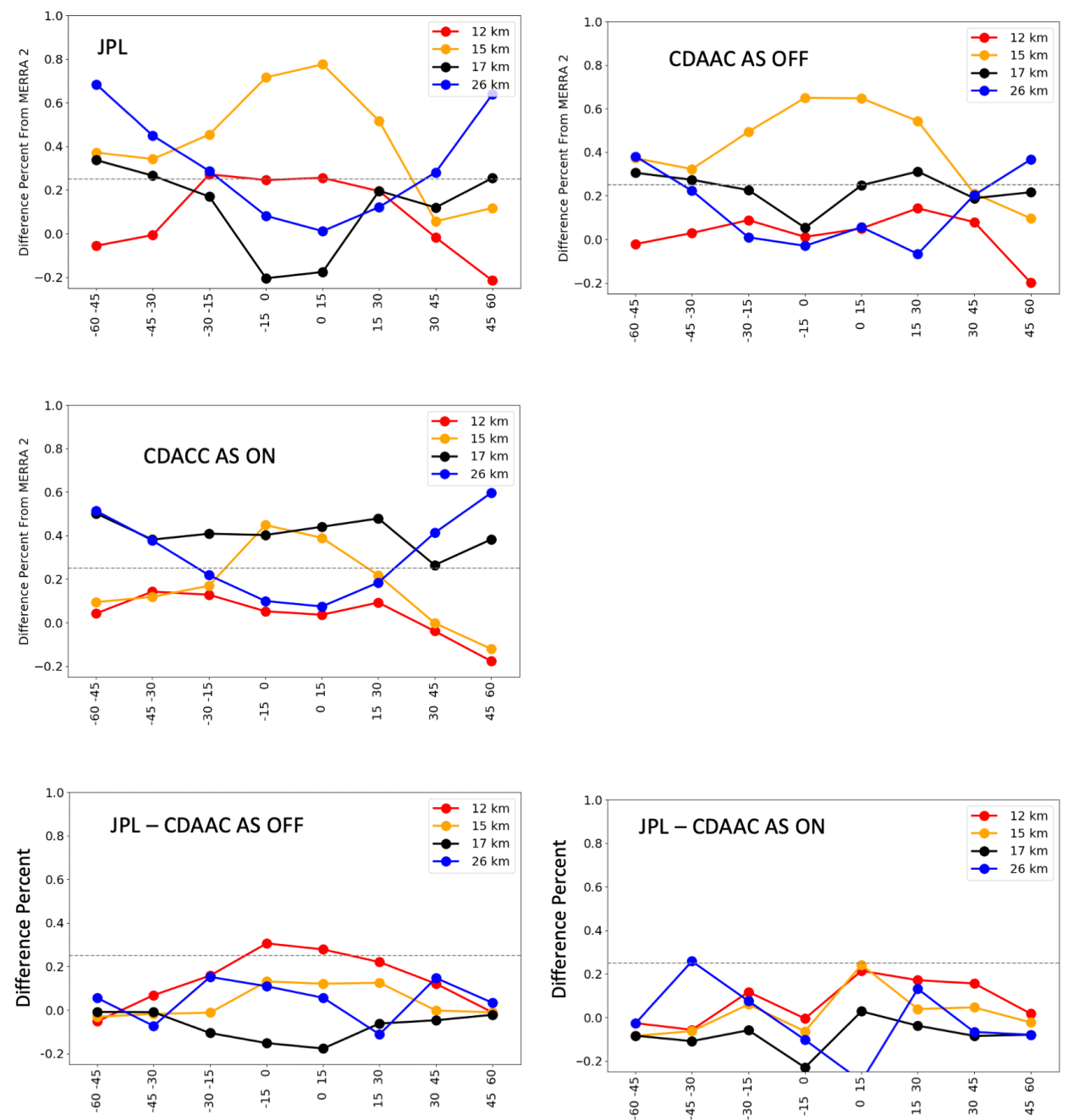

Figure 7. Latitude dependent biases between different data sets for a series of representative altitudes (12, 15, 17 and $26 \mathbf{~ k m}$ ). The top row shows differences of the JPL and CDAAC AS-off data sets minus MERRA-2, for different latitude bands and different altitudes. The middle row shows the differences of CDAAC AS-on minus MERRA-2. The bottom row shows differences of JPL single frequency minus CDAAC AS-on and AS-off data sets, for different latitude bands and altitudes. 